\title{
Haloaldehyde Polymers. I. Chain Termination in Anionic Chloral Polymerization
}

\author{
P. Kubisa* and O. Vogl \\ Polymer Science and Engineering, University of Massachusetts, \\ Amherst, Massachusetts 01002 \\ (Received May 7, 1974)
}

\begin{abstract}
Anionic chloral polymerization can be carried out under certain circumstances in the presence of acylating agents, such as acetyl chloride and benzoyl chloride. Parts of these polymers exhibit a much improved thermal stability. Introduction of acyl end groups into the polymer permits an estimation of the molecular weight of the polymer. DTG was found to be an excellent technique with which to follow the extent of reaction, to assist in the examination of this kind of end group and to follow the thermal stability of chloral polymers.

KEY WORDS Haloaldehyde Polymers / Chloral / Anionic Polymeri-

zation / Chain Transfer / Chain Termination / Acetyl Chloride /

Benzoyl Chloride / Triphenyl Phosphine as Initiator / End Groups /

Thermal Degradation /
\end{abstract}

Our knowledge of the polymerization of chloral has recently been greatly advanced with the development of a new technique to make homoand copolymers of chloral directly in a useful form. $^{1,2}$

Chloral polymerizes rapidly to high conversion with anionic initiators. Cationic polymerization is now also well established, however, it is extremely slow. ${ }^{3}$

Because chloral polymers of high chloral content are insoluble and infusable, much of the recent work on the characterization of these polymers was done by infrared techniques and particularly by the studies of thermal degradation of chloral polymers.

Very little is known about the end groups of chloral polymers, particularly of the homopolymer, although it has been reported that chloral polymers are like the polymers of formaldehyde, substituted polyoxymethylene glycols which can be acetylated to the corresponding acetates. $^{4}$

We found that anionic chloral polymerization can be carried out readily in the presence of strong acylating agents and that acylation occurs in the form of a chain termination reaction. This method provided a new way to introduce

* On leave from Center of Molecular and Macromolecular Studies, Polish Academy of Sciences, Lodz. stable end groups into polychloral. It also provided a method for estimating the minimum number-average molecular weight of the stable polychloral fraction.

\section{EXPERIMENTAL}

\section{Materials}

Chloral (obtained from the Diamond Shamrock Company) was allowed to reflux over phosphorous pentoxide for $24 \mathrm{hr}$. It was then transferred into a carefully dried distillation apparatus which consisted of a $100-\mathrm{cm}$ long column with an electric heating jacket, filled with glass helices of $1 / 2-\mathrm{cm}$ diameter and an automatic distillation head. The distillation was carried out under a reflux ratio of $1: 100$ under purified nitrogen.

The distillation of chloral was carried out continuously. An initial fraction of $20 \%$ was collected which is not useful for the preparation of good chloral polymers. The progress of the distillations was monitored by gas chromatography. Polymerization grade chloral, the main fraction, contains only two impurities: water, less than $100 \mathrm{ppm}$, preferably less than $20 \mathrm{ppm}$ and dichloroacetaldehyde (less than $0.1 \%$ ).

It is not possible to store pure chloral monomer for extended periods. Consequently, the necessary amount of chloral was collected from the distillation apparatus before use. 
Triphenylphosphine (Aldrich) was purified by crystallization from benzene and used as a one molar solution in benzene.

Lithium tertiary butoxide (Alpha Inorganic Company) was purified by vacuum sublimation and used as a one molar solution in methylcyclohexane.

Acetyl chloride, acetyl anhydride, and benzoyl chloride (Eastman Kodak Chemical Company) were purified by distillation.

\section{Polymerization of Chloral}

It was convenient to prepare chloral polymers for various measurements in the form of films. Since the polymer is insoluble and infusable the film must be cast directly from the monomer.

The initiated monomer solution was prepared as follows: A large test tube $(50 \mathrm{ml})$ was flamed out and dried under nitrogen. It was fitted with a serum cap and a nitrogen inlet and outlet and placed into an oil bath at $70^{\circ} \mathrm{C}$. Freshly distilled chloral was transferred from the receiver of the distillation apparatus with a predried hypodermic syringe into the test tube. The test tube was immersed into the oil bath and after 5 min the temperature of the contents of the test tube has reached the temperature of the oil bath. The initiator solution and the additive used as the chain-transfer agent were injected with small syringes and the solution was shaken until it was homogeneous.

The monomer is initiated and all the handling of this solution must now be carried out with equipment which is preheated to at least $65^{\circ} \mathrm{C}$.

The solution from the test tube was then transferred with a hypodermic syringe into the polymerization assembly. This polymerization assembly consisted of two glass plates $(20 \mathrm{~cm} \times$ $20 \mathrm{~cm} \times 0.8 \mathrm{~cm}$ ) which were separated by a strand of elastic fiber and clamped together with simple bureau clamps; the assembly had also been preheated to $70^{\circ} \mathrm{C}$ in order to prevent premature polymerization.

After the initiated monomer solution was filled into the assembly, the whole assembly was immersed immediately into an ice-water bath. After $1 \mathrm{hr}$ the assembly was taken out and allowed to dry at room temperature; this was usually accomplished overnight. The glass plates were then separated, the polymer film was cut to the proper size, extracted with acetone for $48 \mathrm{hr}$ in a Soxhlet extractor and dried under reduced pressure at room temperature for several hours.

\section{Measurements}

All infrared spectra of polychloral films were recorded on a Perkin-Elmer 727 spectrophotometer.

The thickness of different samples of chloral film varied depending on several variables, especially the thickness of the spacer material. Consequently, an internal standard method was used to compare the intensity of the carbonyl absorption of the end groups of chloral polymers.

The peak at $2625 \mathrm{~cm}^{-1}$, which appears in the spectra of pure polychloral was chosen as the standard. The "relative peak intensity" which describes the ratio of the carbonyl peak $\left(1750 \mathrm{~cm}^{-1}\right.$ for the carbonyl group of the benzoyl group or $1780 \mathrm{~cm}^{-1}$ for the acetyl group) to the $2625 \mathrm{~cm}^{-1}$ peak (measured in the absorbance scale) was used as the value for comparison.

To determine quantitatively the dependence of the concentration of the ester end groups on "the relative carbonyl peak intensity," a series of "homogeneous" mixtures of polychloral and poly(methyl methacrylate) with a poly(methyl methacrylate) content of less than $3 \mathrm{~mol} \%$ was prepared by "single phase polymerization." 5 The molar ratio of the components was determined by elemental analysis and the "relative carbonyl peak intensity" of these polymer films were measured.

From these results the concentration of the ester end groups could be calculated assuming that the extinction coefficient in both cases was approximately the same. The previous assumption was justified because it was known that the extinction coefficient of the carbonyl group in acetates was $385-410 \mathrm{~m} \times l \times \mathrm{cm}^{-1}$ and in methacrylates $390-440 \mathrm{~m} \times l \times \mathrm{cm}^{-1}{ }^{6}$

The thermal degradation of chloral polymer samples was determined in nitrogen using a Perkin-Elmer TGS-1 thermobalance at a programmed increase of temperature of $5^{\circ} \mathrm{C} / \mathrm{min}$.

The purity of the monomer was monitored by gas chromatography with a Varian Aerograph Model 920, a column packed with Poropack and 


\section{P. Kubisa and O. Vogl}

helium as the carrier gas.

\section{RESULTS AND DISCUSSION}

The rate of chloral polymerization depends very much on the initiator used. Most initiators fall into two general categories; one category consists of those initiators which initiate by an almost quantitative initiation and the other consists of those which initiate by a very low degree of initiation. Up to now there are no initiators known which have an initiation equilibrium, where $k_{\mathrm{add}} \approx k_{\mathrm{diss}}$; the initiating anion added to an apprreciable amount but not completely to the carbonyl carbon of the chloral carbonyl group.

There is anthor group of initiators which reacts more violently with the monomer and may cause side reactions. However, when treated properly such initiators may also act as good initiators; the addition of these anions to chloral is essentially quantitative, as in the case of butyl lithium.

Of the convenient initiators, lithium tertiary butoxide is preferred because it reacts fast and essentially quantitative with one mole of chloral to form the corresponding alkoxide anion, without causing any side reaction. ${ }^{7}$<smiles>C=C=CCOC(OCC)C(Cl)(Cl)Cl</smiles>

Compounds belonging to the second group of weak initiators are the onium salts, particularly tetraalkylammonium chlorides and tetraalkyl and aryl phosphonium chlorides. ${ }^{2}$ The initiating equilibrium between the chloride ion and chloral monomer is on the left hand side of the equation.<smiles>[O]C(Cl)C(Cl)(Cl)Cl</smiles>

Another group of initiators belongs to the same category but it is not easily recognizable as be- ing part of the same category except that the overall rate of polymerization of chloral with these initiators is very similar to that of onium salts as initiators of chloral polymerization. This group of initiators includes tertiary amines and particularly tertiary phosphines.

In the case of tertiary phosphines, particularly of triphenylphosphine, we have clearly established previously that the initiation reaction proceeds in two steps. $^{2}$ Firstly, an instantaneous reaction of triphenylphosphine occurs with one mole of chloral to form triphenyldichlorovinyloxyphosphonium chloride in a fast and quantitative<smiles>O=C([Pb]OC=C(Cl)Cl)C(Cl)(Cl)c1ccccc1</smiles>

reaction. This chloride is now the real initiator and apparently initiates according to eq 2 and 3.

The nature of the initiator determines the structure of one of the end groups in the polychloral chain. Unfortunately, very little is known about the reaction terminating the growth of the macromolecule. Consequently, the nature of the terminal end groups in chloral polymerizations is not known and infrared studies do not give any indication of a clearly identifiable, specific end group.

In spite of previous reports that chloral polymers can be easily end capped like polymers of formaldehyde ${ }^{4}$ chloral polymers prepared under our conditions could not be acetylated and after exhaustive extraction with acetone, no carbonyl groups could be detected in any of our polymers.

The nature of the end groups is of particular interest because in the case of polyformaldehyde this is the "weak link" which determines the thermal stability of the polymer. As a consequence, the preparation of polychloral with highly stable end groups, either ester or ether end groups, was considered vital for the preparation of thermally stable polychloral.

Endcapping and copolymerizations are two techniques for the preparation of thermally stable polymers when the initial polymer has limited stability. Another technique to introduce thermally stable end groups, controlled chain transfer, has been successfully demon- 
Haloaldehyde Polymers. I.

strated in the case of trioxane polymerization by Enikolopyan. ${ }^{8}$

In our work, potential chain-transfer agents or terminators for the polymerization of chloral, such as acetyl chlorides or anhydrides have been used as the chain-tranfer agent and/or terminators (CTA) with two initiators-triphenylphosphine and lithium tertiary butoxide.

It was surprising that an anionic polymerization with a growing alkoxide anion would be carried out in the presence of strong acetylating agents in up to $2-3-\mathrm{mol} \%$ quantities without immediate chain termination. It was also surprising that lithium tertiary butoxide could be used at all as initiator and polymerization was not inhibited in a system which contained both lithium tertiary butoxide and benzoyl chloride.

Polymerization of Chloral with Triphenylphosphine $\left(\mathrm{Ph}_{3} \mathrm{P} \oplus-\mathrm{O}-\mathrm{CH}=\mathrm{CCl}_{2} \mathrm{Cl} \odot\right)$

The polymerization of chloral initiated by triphenylphosphine was carried out in the presence of acetyl chloride, acetic anhydride and benzoyl chloride as CTA. All the polymers contained acyl groups indicating that chain transfer or termination had occurred during the polymerization. This reaction can be described as a competitive nucleophilic substitution on the carbonyl carbon atom as indicated in eq 4 .

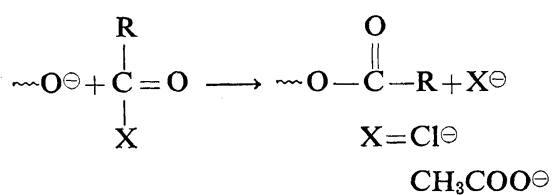

As one would expect, acetyl chloride is the most effective CTA. It gives the highest percentage of stable polymers in the total polymers and it also gives the highest percentage of acetyl end groups in the polymers. Benzoylchloride and acetic anhydride are significantly less effective.

It is interesting to note that the addition of a CTA up to approximately $1 \mathrm{~mol} \%$ does not effect either the rate of polymerization nor the final conversion. Further increase of CTA concentration in the chloral polymerization mixture decreases conversion, probably because of the reaction between CTA and the initiator leading to partial deactivation of the initiator. The results are shown in Tables I, II, and III.
Table I. Bulk polymerization of chloral ${ }^{\text {a }}$ (CTA-acetyl chloride)

\begin{tabular}{ccccc}
\hline No. & $\begin{array}{c}\text { CTA concn, } \\
\text { mol \% }\end{array}$ & $\begin{array}{c}\text { Yield, } \\
\%\end{array}$ & $\begin{array}{c}\text { Rel peak } \\
\text { int. }\end{array}$ & $\begin{array}{c}\text { End-group } \\
\text { concn, mol \% }\end{array}$ \\
\hline $1-0$ & 0 & 81 & 0 & - \\
$1-1$ & 0.22 & 78 & 0.54 & 0.27 \\
$1-2$ & 0.55 & 81 & 0.75 & 0.37 \\
$1-3$ & 1.1 & 81 & 1.10 & 0.55 \\
$1-4$ & 1.65 & 75 & 0.70 & 0.34 \\
$1-5$ & 1.90 & 43 & 0.30 & 0.15 \\
\hline
\end{tabular}

a Initiator, $\mathrm{Ph}_{3} \mathrm{P}, 0.2 \mathrm{~mol} \%$; reaction conditions, $0^{\circ} \mathrm{C}, 1 \mathrm{~h}$, room temp, $16 \mathrm{hr}$.

Table II. Bulk polymerization of chloral ${ }^{\mathrm{a}}$ (CTA-acetic anhydride)

\begin{tabular}{ccccc}
\hline No. & $\begin{array}{c}\text { CTA concn, } \\
\text { mol } \%\end{array}$ & $\begin{array}{c}\text { Yield, } \\
\%\end{array}$ & $\begin{array}{c}\text { Rel peak } \\
\text { int. }\end{array}$ & $\begin{array}{c}\text { End-group } \\
\text { concn, mol } \%\end{array}$ \\
\hline $1-0$ & 0 & 81 & 0 & - \\
$2-1$ & 0.20 & 76 & 0.21 & 0.10 \\
$2-2$ & 0.65 & 72 & 0.13 & 0.06 \\
$2-3$ & 1.50 & 67 & 0.23 & 0.11 \\
\hline
\end{tabular}

a Initiator, $\mathrm{Ph}_{3} \mathrm{P}, 0.2 \mathrm{~mol} \%$; reaction conditions, $0^{\circ} \mathrm{C}, 1 \mathrm{hr}$, room temp, $16 \mathrm{hr}$.

Table III. Bulk polymerization of chlorala (CTA-benzoyl chloride)

\begin{tabular}{ccccc}
\hline No. & $\begin{array}{c}\text { CTA concn, } \\
\text { mol } \%\end{array}$ & $\begin{array}{c}\text { Yield, } \\
\%\end{array}$ & $\begin{array}{c}\text { Rel peak } \\
\text { int. }\end{array}$ & $\begin{array}{c}\text { End-group } \\
\text { concn, mol } \%\end{array}$ \\
\hline $1-0$ & 0 & 81 & 0 & - \\
$3-1$ & 0.17 & 78 & 0.15 & 0.07 \\
$3-2$ & 0.50 & 80 & 0.20 & 0.10 \\
$3-3$ & 0.85 & 78 & 0.34 & 0.17 \\
$3-4$ & 1.20 & 51 & 0.11 & 0.05 \\
\hline
\end{tabular}

a Initiator, $\mathrm{Ph}_{3} \mathrm{P}, 0.2 \mathrm{~mol} \%$; reaction conditions, $0^{\circ} \mathrm{C}, 1 \mathrm{hr}$, room temp, $16 \mathrm{hr}$.

\section{Chloral Polymerization with Lithium Tertiary Butoxide}

Triphenylphosphine (which as indicated before acts as triphenyldichlorovinyloxyphosphonium chloride) was selected as the initiator for the study of CTA in chloral polymerizations because the anion of the phosphonium salt, the chloride, is a very weak nucleophile and cannot be acylated by the acylating agents used as CTA. Benzoyl chloride and acetyl chloride, the preferred CTA's can be viewed as products of 


\section{P. Kubisa and O. VoGL}

acylation of chloride ions. As a consequence, only the acylations of the subsequent alkoxides which are the carriers of the anionic polymerization of chloral must be considered as important intermediates for chain transfer or termination of chloral polymerization with CTA's.

More efficient nucleophiles such as tertiary butoxides not only produced the initiating species ( $1: 1$ addition products with chloral) in essentially quantitative yields but they also may react with the acylating agents, the CTA. A competitive reaction may occur between the addition of lithium tertiary butoxide to the electrophilic carbonyl carbons of chloral as compared to the potentially more electrophilic carbon of the acid chlorides of benzoic acid or acetic acid. Ultimately, because of the possibility of loss of chloride from acid chlorides or of acetate from acetic anhydride, the formation of the tertiary butyl ester was expected to be the final product if equilibrium conditions existed.

The addition of lithium tertiary butoxide to chloral is also an equilibrium reaction although the equilibrium is essentially quantitatively on the side of the addition product.

Experiments showed that this consideration si qualitatively correct. In the presence of the stronger electrophilic acetyl chloride, polymerization of chloral did not occur with lithium tertiary butoxide as the initiator. Less reactive compounds such as acetic anhydride and benzoyl chloride apparently did not compete as effectively with chloral for the lithium tertiary butoxide and polymerization proceeded to relatively high conversion.

Even if the mixture of chloral monomer, the initiator (lithium tertiary butoxide) and benzoyl chloride was held for as much as $10 \mathrm{~min}$ above the threshold temperature of chloral and the polymerization mixture was the cooled to perform the cryotachensic polymerization the conversion of monomer to polymer was still high. Chloral polymerization with lithium tertiary butoxide as initiator as a function of the CTA concentration is shown in Table IV. It can be seen that up to $1 \mathrm{~mol} \%$, of CTA concentration did not effect the polymer yield and the relative carbonyl peaks observed in the polymer. The end group concentration calculated from the
Table IV. Bulk polymerization of chlorala (CTA-benzoyl chloride)

\begin{tabular}{ccccc}
\hline No. & $\begin{array}{c}\text { CTA concn, } \\
\text { mol } \%\end{array}$ & $\begin{array}{c}\text { Yield, } \\
\%\end{array}$ & $\begin{array}{c}\text { Rel peak } \\
\text { int. }\end{array}$ & $\begin{array}{c}\text { End-group } \\
\text { concn, mol \% }\end{array}$ \\
\hline $4-1$ & 0 & 34 & - & - \\
$4-2$ & 0.08 & 58 & 0.18 & 0.09 \\
$4-3$ & 0.25 & 48 & 0.19 & 0.09 \\
$4-4$ & 0.34 & 48 & 0.13 & 0.06 \\
$4-5$ & 0.42 & 68 & 0.18 & 0.09 \\
$4-6$ & 0.7 & 35 & - & - \\
$4-7$ & 1.0 & 20 & - & - \\
$4-8$ & 3.4 & 9.5 & - & - \\
\hline
\end{tabular}

a Initiator, $\mathrm{LiOtBu}, 0.2 \mathrm{~mol} \%$; reaction conditions, $0^{\circ} \mathrm{C}, 1 \mathrm{hr}$, room temp, $16 \mathrm{hr}$.

carbonyl peaks indicated that the molecular weight of the polymer was approximately the same. In all cases, where the CTA concentration was increased to more than $3 \mathrm{~mol} \%$, the yield of polymer dropped rapidly.

With acetic anhydride as CTA, the yield of chloral polymer was not effected substantially up to approximately $1-\mathrm{mol} \%$ CTA but dropped significantly at higher CTA concentration (Table V). Table VI shows the dependence of reaction times of the initiated monomer mixture (up to

Table V. Bulk polymerization of chloral $^{\mathrm{a}}$ (CTA-acetic anhydride)

\begin{tabular}{ccc}
\hline No. & CTA concn, mol \% & Yield, \% \\
\hline $5-1$ & 0.22 & 46 \\
$5-2$ & 0.44 & 39 \\
$5-3$ & 0.66 & 35 \\
$5-4$ & 0.88 & 22 \\
\hline
\end{tabular}

a Initiator, $\mathrm{LiOtBu}, 0.2 \mathrm{~mol} \%$; reaction conditions, $0^{\circ} \mathrm{C}, 1 \mathrm{hr}$, room temp, $16 \mathrm{hr}$.

Table VI. Bulk polymerization of chlora1 ${ }^{\mathrm{a}}$ ${(\text { CTA-benzoyl chloride })^{b}}^{b}$

\begin{tabular}{ccc}
\hline No. & Time $\left(\right.$ at $\left.70^{\circ} \mathrm{C}\right)$ & Yield, $\%$ \\
\hline $6-1$ & $10 \mathrm{sec}$ & 55 \\
$6-2$ & $3 \mathrm{~min}$ & 66 \\
$6-3$ & $6 \mathrm{~min}$ & 59 \\
$6-4$ & $10 \mathrm{~min}$ & 42 \\
\hline
\end{tabular}

a Initiator, $\mathrm{LiOtBu}, 0.2 \mathrm{~mol} \%$; reaction conditions, $0^{\circ} \mathrm{C}, 1 \mathrm{hr}$, room temp, $16 \mathrm{hr}$.

b $0.34 \mathrm{~mol} \%$. 
$10 \mathrm{~min}$ ) in the presence of $0.34 \mathrm{~mol} \%$ of CTA, which is twice the amount of the initiator used for initiating this polymerization, on the polymer yield. The yield is not changed up to a $10-\mathrm{min}$ initial reaction.

The yields of chloral polymers initiated with lithium tertiary butoxide (Table IV) are lower than the corresponding yields of polymers initiated with triphenyl phosphine (Tables I, III). These yields are not the original yield of monomers to polymer, but the yields after $48-\mathrm{hr}$ extraction with acetone; the actual yield of monomer to polymer is always $80-85 \%$; the reduction in yield after extraction reflects the instability of the polychloral prepared with this initiator system and the degradation of the "unstable fraction" during the extraction.

As indicated above, the initiation of chloral polymerization with lithium tertiary butoxide involved the fast and essentially quantitative formation of the alkoxide ion as indicated in eq 5 .

$$
\begin{aligned}
& \left(\mathrm{CH}_{3}\right)_{3} \mathrm{C}-\mathrm{O} \ominus \mathrm{Li} \oplus+\underset{+}{\mathrm{C}}=\mathrm{CC}{ }_{3} \\
& \longrightarrow\left(\mathrm{CH}_{3}\right)_{3} \mathrm{C}-\mathrm{O}-\underset{\mathrm{I}}{\mathrm{C}}-\mathrm{Cl} \mathrm{C}_{3}
\end{aligned}
$$

In the presence of acetyl chloride, an other competitive reaction can occur. (eq 6).

$$
\begin{aligned}
\left(\mathrm{CH}_{3}\right)_{3} \mathrm{C}-\mathrm{O} \ominus \mathrm{Li} \oplus+\mathrm{RCOCl} & \\
& \longrightarrow\left(\mathrm{CH}_{3}\right)_{3} \mathrm{C}-\mathrm{O}-\mathrm{CO}-\mathrm{R}+\mathrm{Li} \oplus \mathrm{Cl} \ominus
\end{aligned}
$$

When the highly reactive acetyl chloride was used as a CTA, reaction 6 proceeded fast and all the initiator was transformed into unreactive esters, before polymerization could occur. We have not determined whether this unreactive ester was the tertiary butyl ester of acetic acid or the reation product of the lithium tertiary butoxide with chloral which was then acetylated by acetyl chloride. ${ }^{7}$ Should the first case be

$$
\begin{gathered}
\mathrm{CCl}_{3} \stackrel{\mathrm{O}}{!} \\
\left(\mathrm{CH}_{3}\right)_{3} \mathrm{C}-\mathrm{O}-\mathrm{C}-\mathrm{O}-\mathrm{C}-\mathrm{CH}_{3}
\end{gathered}
$$

correct, two possibilities would account for our results. The first possibility is the direct reaction of acetyl chloride with lithium tertiary butoxide before its addition to chloral can occur. The second possibility is that lithium tertiary butoxide added initially to chloral but then acetyl chloride reacted with the small amount of lithium tertiary butoxide which is in equilibrium with the alkoxide addition product. Ultimately the $1: 1$ adduct undergoes completely the reverse reaction and tertiary butoxide reacts completely with acetyl chloride to form tertiary butyl acetate.

Benzoyl chloride is a much weaker nucleophile and apparently cannot compete when a large excess of monomer is present. The relatively small influence of time of initiation (between mixing and cooling) on the conversion of this polymerization can be explained on the basis of relatively low reactivity of the new alkoxide ion with benzoyl chloride as compared to the tertiary butoxide anion. This is caused by the strong inductive effect of the trichloromethyl group of the new alkoxide.

The yields of polymerization given in Tables I-IV were determined after 48-hr extraction with acetone. The relatively low yield of polymerization with $\mathrm{LiO}-t \mathrm{Bu}$ as initiator was due to the partial decomposition of polymer during extraction. An extreme example of instability of polychloral was the complete decomposition of polymers prepared with quarternary ammonium salts as initiators $\left(\mathrm{Et}_{4} \mathrm{~N} \oplus \mathrm{Cl} \ominus\right.$ and $\mathrm{Bu}_{4}{ }^{\oplus} \mathrm{NCl} \ominus$ ) during extraction. In Table VII the yields of polymerization determined by two different methods are compared. The yield before extraction was determined by removing unreacted monomer from polymer films in vacuum $(1 \mathrm{mmHg})$ at room temperature for $24 \mathrm{hr}$.

Table VII. Bulk polymerization of chloral ${ }^{\mathrm{a}}$

\begin{tabular}{lccc}
\hline Initiator & CTA & $\begin{array}{c}\text { Yield before } \\
\text { extr. }\end{array}$ & $\begin{array}{c}\text { Yield after } \\
\text { extr. }\end{array}$ \\
\hline $\mathrm{Ph}_{3} \mathrm{P}$ & - & & 81 \\
$\mathrm{Ph}_{3} \mathrm{P}$ & $\mathrm{CH}_{3} \mathrm{COCl}$ & & 81 \\
$\mathrm{LiOtBu}$ & - & 85 & 34 \\
$\mathrm{LiOtBu}$ & $\mathrm{C}_{6} \mathrm{H}_{5} \mathrm{COCl}$ & 85 & 68 \\
$\mathrm{R}_{4} \mathrm{~N} \oplus \mathrm{Cl} \ominus$ & & 86 & 00 \\
$\mathrm{R}_{4} \mathrm{~N} \oplus \mathrm{Cl} \ominus$ & $\mathrm{CH}_{3} \mathrm{COCl}$ & 37 & Low, irre- \\
& & & producible \\
\hline
\end{tabular}

a Initiator concn, $0.2 \mathrm{~mol} \%$; reaction conditions, $0^{\circ} \mathrm{C}, 1 \mathrm{hr}$, room temp, $16 \mathrm{hr}$. 
In all cases, however, the IR spectrum showed the presence of small amounts of monomer remaining in the polymer samples; besides, the polymer still contained initiator fragments. Consequently, this method cannot be used, when the pure polymer is needed.

At the present time we cannot fully explain all our results, nor can we decide whether the different stability of the polymer samples are due to the different reactivities of the polymer ends or to the different abilities of the remaining initiator (or side products) to catalyze a decomposition of polymer.

Polychloral is not soluble in any known solvent. Earlier investigators have mentioned basic solvents, most prominently pyridine, as a solvent for polychloral. ${ }^{9}$ We have investigated this work very carefully and found that pyridine as well as other amines do not dissolve polychloral without complete degradation of the polymer to monomer. We have followed this degradation by NMR and found that the NMR spectrum of the solution contains only one peak characteristic for chloral monomer. No peak was found in the NMR especially in the range of $5-7 \mathrm{ppm}$ where the acetalic protons are expected to show. ${ }^{10}$ In addition, no polychloral could be precipitated from the pyridine "solution." It should also be pointed out that only some polychloral samples are degraded by pyridine. Well stabilized samples of polychloral cannot be dissolved nor do they degrade in pyridine.

Under these circumstances, the determination of the degree of polymerization of polychloral by a conventional method based on solution properties is not possible. Methods based on end-group analysis have been used successfully and routinely for the determination of the number-average molecular weight of polyformaldehyde. $^{11}$

There is also a suggestion in the literature that this method has been successfully used for the determination of molecular weights of polychloral. ${ }^{4}$ In our hands, polychloral once made and isolated, cannot be endcapped, no trace of carbonyl group is observed after acetylation with acetic anhydride or acetyl chloride and exhaustive extraction of the polymer sample with acetone.

A method to determine the degree of polymerization of polychloral based on end-group analysis of acyl groups is correct only in the case when: (a) There is positive evidence that the terminating agent (or chain-transfer agent) reacted with the polymer and not with impurities (monomer or initiator) and consequently, that the compound which contains the acetyl group is chemically bound to the mocromolecule: (b) There is positive evidence that all macromolecules contain the type of end group for which an analysis can be carried out, or the fraction of the total polymer containinig those particular end groups is known. Otherwise the molecular weight estimation will only give a maximum number-average molecular weight.

The first condition is fulfilled in this case of polychloral. Infrared spectra of polychloral films (Figure 1) prepared with triphenylphosphine

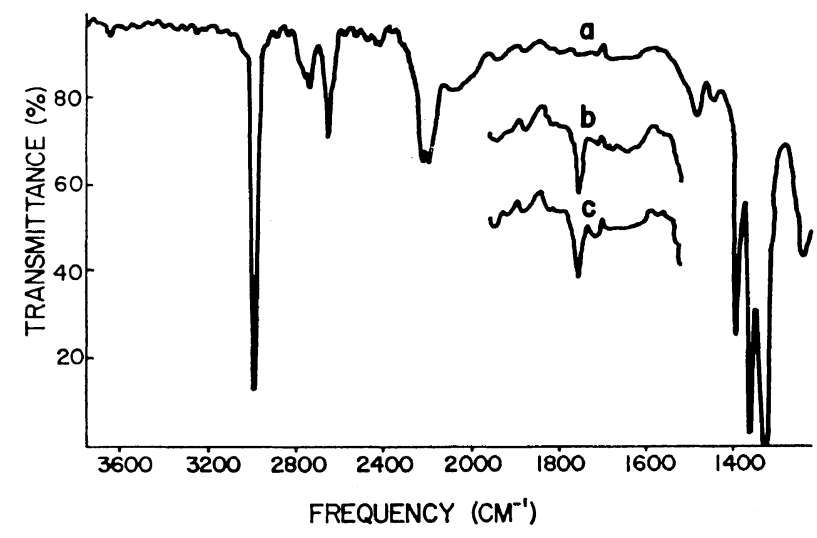

Figure 1. IR spectra (film) of polychloral: End group identification. 
as initiator in the absence of CTA, showed no absorption in the spectra of the polymer film in the range of $1700-1800 \mathrm{~cm}^{-1}$ which would be characteristic for carbonyl bonds after a $48 \mathrm{hr}$ extraction with acetone (Figure $1 \mathrm{a}$ ). The complete absence of a carbonyl absorption in this region indicated that no unreactive monomor is present in the polymer.

Elemental analysis showed that polychloral is free from traces of initiator. Chloral polymer initiated with $0.2 \mathrm{~mol} \%$ triphenylphosphine should have a phosphorous content of $0.080-\%$ phosphorous in the polymer assuming 100-\% conversion. After extraction for $48 \mathrm{hr}$ with acetone, typical analysis of chloral polymers for phosphorous showed that less than $0.002 \%$ of phosphorous remained in the polymer $(<3 \%$, accuracy of $P$ analysis $\pm 0.002 \%$ ).

Infrared spectra of chloral polymers prepared in the presence of CTA showed the existence of carbonyl bonds in the polychloral. The intensity of the absorption did not change after an additional $48 \mathrm{hr}$ of acetone extraction (Figure $1 \mathrm{~b}, \mathrm{c})$ which indicated that the carbonyl group is part of the ester group which is chemically bound to the polychloral.

In the course of our work on the thermal behavior of polychloral, we found that the thermal stability depended greatly on the nature

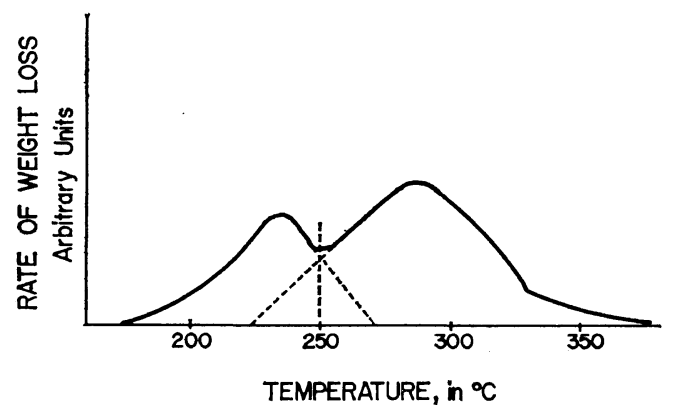

Figure 2. Polychloral: DTG curve of polymer prepared in the presence of acetylating agents. of the polymer end groups. ${ }^{12}$ In Figure 2 the differential thermogravimetric analysis curve (DTG) for the decomposition of polychloral prepared in the presence of acetyl chloride with triphenylphosphine as initiator is shown. The existence of two maxima in the curve indicates that polychloral obtained under these conditions is a mixture of two fractions. Only the more stable fraction, which has a maximum at $285^{\circ} \mathrm{C}$ contains ester and groups and is described as $\%$ "stable fraction" in Table VIII.

The amount of this fraction can be approximated by measuring the surface under the corresponding part of the curve and comparing its amount with the total area under the curve.

Using this approximation, the degree of polymerization of the stable fraction, the portion of the polymer which contains one ester end group, can now be calculated from the concentration of ester end groups (Table I, 1-2, Table II, 2-2, Table III, 3-3) in the total polymer.

A specific calculation for the DP of the stable fraction of (1-2) polychloral is as follows:

$$
\begin{aligned}
\mathrm{DP}= & (100 \mathrm{~mol} \% \text { of end groups }) \\
& \times(\% \text { of stable fraction } / 100) \\
= & (100 / 0.37) \times(70 / 100)=189
\end{aligned}
$$

Table VIII shows the results of these approximations. They should be treated only as approximate values and are probably not more accurate than $\pm 5 \%$ in "stable fraction."

In Table VIII, the DP of polymers prepared with different CTA concentration are compared; this was necessary because it was not possible in every case to calculate with sufficient accuracy the percentage of "stable fraction." Nevertheless, it was clear that the differences in the endgroups concentration were due to the different percentage of the polymer-containg ester end groups and not to the different degree of polymerization, which was almost the same in all cases. It also showed that the chain-transfer

Table VIII.

\begin{tabular}{ccccc}
\hline No. & CTA & CTA concn, mol \% & \% of "stable fraction" & DP of "stable fraction" \\
\hline $1-2$ & $\mathrm{CH}_{3} \mathrm{COCl}$ & 0.55 & 70 & 189 \\
$2-2$ & $\left(\mathrm{CH}_{3} \mathrm{CO}\right)_{2}$ & 0.65 & 13 & 217 \\
$3-3$ & $\mathrm{C}_{6} \mathrm{H}_{5} \mathrm{COCl}$ & 0.85 & 35 & 206 \\
\hline
\end{tabular}




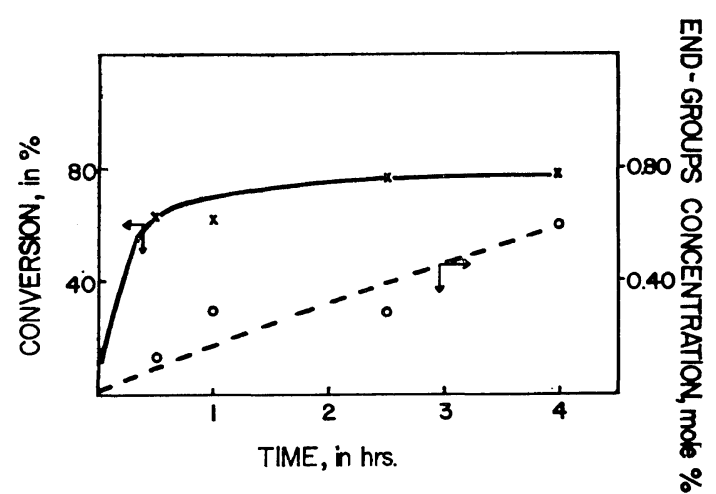

Figure 3. Polychloral: Progress of acetylation during polymerization.

reaction was relatively slow in comparison to the propagation reaction. (Figure 3) Results shown in Figure 3 indicate that at $60-\%$ conversion to polymer, the concentration of ester end groups was low, it increased continuously after the polymerization was practically complete.

To explain these results the following scheme can be proposed: During the first period of the reaction (up to 70-\% conversion) propagation occurs, which is accompanied by only very limited chain transfer reaction $\left(k_{\mathrm{p}} \cdot[\mathrm{M}] \gg k_{\mathrm{tr}} \cdot[\mathrm{CTA}]\right)$. In the next period, when the polymerization was close to equilibrium conditions (last 10\% of conversion), the propagation was very slow, but the growing anions were still living and chain-transfer reaction proceeded, since almost all of the monomer was already polymerized, reinitiation did not occur to any significant extent.

The whole process can be described as a polymerization with following "endcapping" reaction, which in this case was effective because the "endcapping" agent (CTA) was uniformly distributed inside the polymer network and in close proximity to the growing (living) polymeric anion.
According to this scheme, the degree of polymerization of the "stable fraction" should be very close to the degree of polymerization of not-endcapped polymers. Consequently, the numbers given in Table VIII characterize the degree of polymerization $\left(\bar{M}_{n}\right)$ of the whole sample.

Polymerization of chloral in the presence of acid chlorides and anhydrides provideds a simple method to estimate the degree of polymerization of polychloral. It also provided a lead to a possible simple method for the preparation of highly stable chloral homopolymers.

Acknowledgement. This work was supported by the National Science Foundation. One of us (P.K.) would like to thank the Polish Academy of Sciences for the permission to study abroad.

\section{REFERENCES}

1. O. Vog1, U.S. Patent 3454527 (1969).

2. O. Vog1, U.S. Patent 3668184 (1972).

3. O. Vogl and P. Kubisa, Visokomol. Soedin., Part II, in press.

4. J. Rosen, C. L. Sturn, G. H. McCain, R. M. Wilhjelm, and D. E. Hudgin, J. Polym. Sci., Part A, 3, 1535 (1965).

5. O. Vog1, U. S. Patent 3707524 (1972).

6. A. R. Katricky, J. M. Lagowski, and J. A. T. Beard, Spectrochim. Acta, 16, 954 (1960).

7. O. Vog1, unpublished results.

8. N. I. Vasilev, V. I. Irzhak, G. F. Telegin, and N. S. Enikolopyan, Dokl. Akad. Nauk, SSSR, 176, 831 (1967).

9. D. E. Ilyina, B. A. Krentzel, and G. E. Semenido, J. Polym. Sci., Part C, 4, 999 (1964).

10. E. G. Brame, Jr. R. S. Sudol, and O. Vog1, ibid., Part A, 2, 5337 (1964).

11. E. I. du Pont de Nemours Co., British Patent 770717 (1957), French Patent 1131939 (1956).

12. P. Kubisa and $\mathrm{O}$. Vogl, in preparation. 